\title{
In Vito Fertilization in Buffaloes: A Review
}

\author{
Saber Mohamed Abd-Allah*
}

Theriogenology Department, Faculty of Veterinary Medicine, Beni-Suef University, Beni-Suef 62511, Egypt

\begin{abstract}
This is the review of original data concerning the effect of some factors on oocyte development in vitro of buffaloes. In vitro fertilization is a multi - step process: oocytes maturation, fertilization and embryo culture.

In vitro fertilization is strongly influenced by events occurring during oocyte maturation, fertilization and the subsequent development of the fertilized oocytes. With the advancement of IVF procedures, variability in developmental rate and viability of in vitro produced buffalo embryos so, improving the efficiency and identifying the sources of variations between IVF systems are more important when routinely producing blastocysts from individuals of high genetic merits. Also, the development of specific culture regimes capable of supporting in vitro maturation (IVM), in vitro fertilization (IVF) and in vitro culture (IVC) to the blastocyst stage is highly desirable in breeding systems. This paper discusses the technical aspects of the procedures involved in in vitro fertilization of buffaloes.
\end{abstract}

Keywords: In vitro, buffaloes, fertilization, IVF.

\section{INTRODUCTION}

For several years, there has been considerable interest in the application of reproductive technologies in buffaloes, both for research and improved productivity. As commercial buffalo's production has expanded, reproductive technologies that are used in cattle (IVF) have been attempted in buffaloes. Although similarities between cattle and buffaloes have facilitated the direct application of these technologies to the latter, critical differences in biology and husbandry have limited application and success.

A practical IVP technique is a key factor in commercial embryo production and transfer technology and offers the opportunity to implement novel animal breeding and production programs

In vitro fertilization is a multi - step process: oocytes maturation, fertilization and embryo culture. Oocyte maturation is the first and the most critical step towards successful in vitro fertilization. In vitro maturation (IVM) of oocytes provide an excellent opportunity for cheap and abundant embryos for carrying out basic research and for the application of emerging biotechnologies like cloning and transgenic [1].

Abd-Allah (2003, 2011a and b) [2-4] had studied different aspects of IVM in buffalo oocytes. The maturation media with the selection of protein supplements and hormones play an important role for IVM in and subsequent for IVF and in vitro development. In most studies on IVM of animal

*Address correspondence to this author at the Theriogenology Department, Faculty of Veterinary Medicine, Beni-Suef University, Beni-Suef 62511, Egypt; Tel: +201114822689; Fax: +20822327982;

E-mail: abdallahsaber49@gmail.com oocytes, the basic medium is supplemented with different kinds of sera [2-5]. The importance of sera may due to its contents of hormones, trace nutrients and proteins such as globulin and futuin [6]. The addition of serum to the culture medium provides a source of albumin that balances the osmolarity and acts as a free radical scavenger [7]. Also, in some experiments, the protein additives of the same species were utilized for in vitro maturation of their occytes. Examples of such protein additives were estrous goat serum for caprine occytes [8], water buffalo follicular fluid for buffalo oocytes [9], porcine follicular fluid for porcine oocytes [10], and equine follicular fluid for equine oocytes [11].

Buffalo oocytes have been studied for different aspects of maturation [2]. In all experiments, maturation media was supplemented with FCS.

This paper discusses the technical aspects of the procedures involved in in vitro fertilization.

\section{COLLECTION OF OVARIES}

For purposes of genetic improvement, ovaries must be collected from genetically superior breeds. Ovaries are collected within 10 minutes after the slaughter of the animals, and kept in a sealed container containing physiological saline $(0.9 \% \mathrm{NaC} 1$ with $100 \mu \mathrm{m} / \mathrm{ml}$ Streptomycin and $100 \mathrm{IU} / \mathrm{ml}$ Penicillin), at a temperature ranging from $28-33^{\circ} \mathrm{C}$.

In other reports, storage at $20^{\circ} \mathrm{C}$ is preferable, but in our experience, such low storage temperatures can reduce the development potential of the oocytes. Careful observation of the temperature of the saline is important to maintain the viability of the ovaries during the period of collection. 
The question of the time interval between animal slaughter and oocyte recovery from the ovaries and the temperature of the holding medium

is an important one. In general, if the time spent from ovary collection to oocyte recovery is short, 30 $33^{\circ} \mathrm{C}$ temperature of the physiological saline is better. However, in cases where the slaughterhouse is distant from the laboratory, a decrease in the temperature is recommended, but the temperature should still be kept above $28^{\circ} \mathrm{C}$ to maintain the development potential of the oocytes. Prolonging the period of ovary collection may significantly affect the viability of the oocytes for in vitro maturation. It is recommended that oocytes should be collected from the ovaries within a 6-hour period. Beyond six hours, development potential is greatly compromised

\section{OOCYTE RECOVERY}

Before oocyte recovery, it is necessary to wash the ovaries in fresh, sterile physiological saline (without antibiotics) to further remove any contaminants. Briefly rinsing the ovary in $70 \%$ ethyl alcohol is recommended, to eliminate surface organisms before processing begins. The ovaries are dried lightly with sterile paper towels before primary oocytes are recovered from 2-8 $\mathrm{mm}$ vesicular follicles to avoid contaminating the aspirates during oocyte retrieval. It is important to remove the big follicles (beyond $9 \mathrm{~mm}$ ) because they contain secretions that cause jelly formation in the aspirates.

Ovaries were collected from slaughtered buffaloes, the aspiration method is commonly employed because of the convenience associated with its application. Aspiration of oocytes is done using a needle attached to a $10-\mathrm{ml}$ syringe. To avoid disruption of the surrounding cumulus cells, an 18-gauge needle is used. Possible toxicity associated with syringes containing rubber plungers and siloxane lubricants is avoided by washing and sterilizing glass syringes under the stringent conditions used for tissue culture glassware. For livestock, the use of plastic disposable syringes is acceptable.

One of the difficulties associated with the aspiration approach lies in the fact that oocytes may only be retrieved from some $30-60 \%$ of the punctured follicles. To minimize this effect, it is necessary to prime the needle and syringe with approximately $0.25-0.5 \mathrm{ml}$ of aspiration medium. This is to provide the initial fluid that helps ensure the retrieval of the follicular contents, especially in collecting the first few oocytes.
The common aspiration medium used is modified phosphate-buffered saline with $3 \%(\mathrm{v} / \mathrm{v})$ inactivated calf serum. Aspiration medium can be prepared once, stored in a refrigerator and made available for one week's activity. However, it is very important to prewarm the aspiration medium up to $38^{\circ} \mathrm{C}$ before use. Alternatively, Medium-199 with Earle's salts, Lglutamine, $25 \mathrm{mM}$ HEPES, supplemented with $3 \%(\mathrm{v} / \mathrm{v})$ inactivated calf serum (heat-treated at $56^{\circ} \mathrm{C}$ for $30 \mathrm{~min}$ ) and trace amounts of antibiotics, can also be used.

After aspiration, the contents of the syringe are slowly dispelled into a sterile centrifuge or test tube with minimum disruption of the cumulus oocyte complex (COC). Once the last ovary of a particular batch is processed, the oocytes are allowed to settle to the bottom of the tube for at least 5 minutes. The precipitate is taken using a sterilized Pasteur pipette and poured into sterile Petri dishes for subsequent searching of COCs. As much as possible, recovery work must be done in a sterile environment with a room temperature of $25^{\circ} \mathrm{C}$.

Sometimes the ovaries are sliced in order to retrieve oocytes. In fact, this method enhances the retrieval of a larger number of oocytes. However, it requires a longer period of retrieval and is not advisable when manipulating large number of ovaries because the extended period of oocyte retrieval would lower the in vitro maturation potential of oocytes.

The third method used for slaughtered animals is follicle dissection. This method is also effective in retrieving good-quality oocytes. However, it may also increase the time needed for the retrieval process.

\section{SELECTION OF OOCYTES FOR IN VITRO MATURATION}

After the ovaries have been obtained, they are searched for the oocytes. Once located in the Petri dish, oocytes are lifted in a sterile glass pipette with a bore diameter of about $400 \mu \mathrm{m}$ and transferred into a dish of fresh pre-warmed washing medium (this may be the same as the aspiration medium). The glass pipette used in holding oocytes must have a bore diameter wide enough to avoid disruption of the cumulus cells surrounding the oocytes. The presence of cumulus cells surrounding the immature oocytes is a requisite for successful maturation in vitro, so they must be protected during oocyte retrieval.

Oocytes with compact multilayered cumulus investment and evenly granulated cytoplasm are 
selected for in vitro maturation. It should be noted, however, that the collected oocytes may have a different appearance. Some are denuded (no cumulus cells attached), some are partly denuded, others have swollen, expanded or spider-like cumulus cells, while others have deformed ooplasm. These oocytes should not be used, because they lack the potential to undergo normal maturation and will eventually end as degenerating oocytes after in vitro fertilization.

\section{IN VITRO MATURATION}

Prior to transfer to an in vitro maturation medium, selected oocytes must be washed four times in fresh pre-warmed aspiration medium and subjected to a final wash in in vitro maturation medium before the in vitro maturation droplets are pooled. The oocytes must be free from any contaminants if successful in vitro maturation is to take place.

The medium used for in vitro maturation of oocytes varies among laboratories. It should be considered that the culture medium employed in IVM may affect the proportion of oocytes that reach metaphase II and become capable of undergoing fertilization. It also influences subsequent embryonic development. The culture media employed in maturation of oocytes in vitro can be broadly divided into simple and complex.

Simple media are usually bicarbonate buffered systems containing basic physiological saline with pyruvate, lactate and glucose. The main differences between various types of simple media lie in differences in their ion concentration, and in the concentration of the energy sources. The media are generally supplemented with serum or albumin, with trace amounts of antibiotics added (penicillin, streptomycin, gentamicin). Complex media, on the other hand, contain, in addition to the components of the simple media, amino acids, vitamins, purines and other substances, mainly at levels at which they are found in serum. Fixed nitrogen is present as free amino acids.

The most widely used complex media for IVM of cattle and buffalo oocytes is Tissue Culture Medium 199 (TAC-199) with Earle's salt, LGlutamine and 25 mM HEPES supplemented with $10-20 \%$ heat inactivated serum (be it a fetal calf serum, calf serum, steer serum or an estrus cow serum). Other culture media used are Ham's F-10, MEM, IVMD 101, RPMI and Synthetic Bovine Oviductal Fluid medium and others. All these however, require further supplements in order to promote maturation.
During IVM, extensive redistribution of intracellular organelles occurs. Mitochondria migrate to occupy a perinuclear location, and the cortical granules migrate outward to lie just beneath the vitelline membrane. At all times, there is a need to realize that the crucial test of successful maturation is the ability of the oocytes to undergo normal embryonic development after sperm penetration and fertilization [4].

\section{SPERM CAPACITATION}

To enhance successful fertilization of the oocytes, sperm cells must be motile, have the ability to undergo capacitation and express the acrosome reaction. Sperm must possess the capacity to bind to the zona pellucida and viteline membrane by acquiring the correct binding proteins during maturation, and exposing these binding sites to the oocyte at the appropriate time. They must be incorporated into the oocyte. The sperm acquire many of these capabilities as they pass through the epididymis.

Capacitation of the sperm involves a complex series of biochemical and physiological reactions. It is believed that an important part of the capacitation process is the gradual removal or alteration of the protective coat from the sperm surface, especially in the region of the acrosome. The removal or alteration of this coat permits exposure of receptor sites, allowing sperm to interact with oocyte receptors.

In vitro capacitation procedures are aimed at stimulating the sequence of events that normally occur in the cow's reproductive tract. This involves the removal of seminal proteins and other substances that coat the sperm membrane of the ejaculated semen. To do this, sperm cells are subjected to thorough washing and exposure in media with elevated ionic strength.

\section{IN VITRO FERTILIZATION}

Successful IVF requires appropriate preparation of sperm and oocyte, as well as culture conditions that are favorable to the metabolic activity of the male and female gametes. In buffalo, IVF of in vitro matured oocytes is usually carried out after $23-24$ hours. This is because the peak of oocytes at metaphase II stage is at this period. A $100 \mu \mathrm{l}$ microdroplets of $\mathrm{BO}$ medium containing caffeine, heparin and BSA with $10 \times 106$ sperm $/ \mathrm{ml}$ is prepared to accommodate 10 oocytes.

This is because 1-oocyte/10 $\mu \mathrm{l}$ is an optimum proportion for a successful in vitro fertilization of oocytes. Co-culture of sperm-oocyte for in vitro 
fertilization was done in a humidified environment with $5 \% \mathrm{CO}_{2}$ and $38.9^{\circ} \mathrm{C}$ temperature for 6 to 7 hours. The incubation period was determined by the fact that sperm capacitation inside the female reproductive tract occurred for about 6 hours.

Before in vitro matured oocytes are subjected to IVF microdroplets, they are first partially denuded of the surrounding cumulus cells to allow easy penetration of the sperm cells. They are washed twice in pre-warmed IVF medium to maintain the defined component of the IVF medium.

\section{EMBRYO CULTURE}

The presence of a somatic cell monolayer in the culture medium during in vitro culture of the developing embryo was found to be very important in enhancing its development potential. The provision of cell basement support provides the developing zygotes with a comfortable environment, and secretes some growth factors supportive to further development in vitro. In our system, the cumulus cells that were detached from the cultured oocytes were kept, and the same culture droplets were used for the culture of embryo after in vitro fertilization. However, the culture medium was replaced with a fresh one (pre-incubated for at least 2 hours inside the incubator) prior to the transfer of the embryo. This was to remove any metabolic waste resultant during cumulus-oocyte complex maturation, and provide the embryos with a fresh medium that could sustain their metabolic requirements.

After the sperm-oocyte co-culture and prior to transfer to the in vitro culture droplets, oocytes are washed four times in a washing medium (culture medium) to remove the excess sperm cells attached to or surrounding the zona pellucida. It is important to remove excess sperm cells, to avoid the presence of denaturating cells that may contaminate the components and eventually the efficiency of theculture medium. Therefore, oocytes are transferred to the in vitro culture droplets for further development.
Embryo culture is done inside a water-jacketed incubator with $5 \% \quad \mathrm{CO}_{2}$ at $38^{\circ} \mathrm{C}$ humidified air. Observation of the cleavage rate is done approximately 24 hours after the initiation of in vitro culture. Observation on the development of pre-implantation embryo is done up to 8 to 10 days of in vitro culture.

\section{REFERENCES}

[1] Li Z, Sun X, Chen J, Liu X, Wisely SM, Zhou Q, Renard JPM, Leno GH, Engelhardt JF. Cloned ferrets produced by somatic cell nuclear transfer. Dev Biol 2006; 293(2): 439-448. http://dx.doi.org/10.1016/j.ydbio.2006.02.016

[2] Abd-Allah SM. In vitro fertilization, processing and cryopreservation of buffalo oocytes and embryos. Ph.D. Thesis, Theriogenology, Faculty of Veterinary medicine, Cairo University (Beni-Suef branch) 2003.

[3] Abd-Allah SM. Laboratory production of buffalo embryos. $1^{\text {ST }}$ Ed., LAP-Publishing House, Germany 2011; pp. 217.

[4] Abd-Allah SM. Mini-Straw Culture System - A Novel Method for Buffalo Oocytes Culture. Theriognol Insight 2011; 1(1): 16.

[5] Motlagh MK, Shahneh AZ, Daliri M, Kohram H, Ghragozlou F. In vitro maturation of sheep oocytes in different concentrations of mare serum. Afr J Biotechnol 2008; 7(18): 3380-3382.

[6] Hsu CJ, Holmes SD, Hammond JM. Ovarian epidermal growth factor-like activity. Concentrations in porcine follicular fluid during follicular enlargement. Biochem Biophys Res Commun 1987; 147: 242-247. http://dx.doi.org/10.1016/S0006-291X(87)80112-2

[7] Thompson JG. In vitro culture and embryo metabolism of cattle and sheep embryos - a decade of achievement. Anim Reprod Sci 2000; 30: 273-280.

[8] Keskintepe L, Darwish GM, Kenimer AT, Brackett BG. Term development of caprine embryos derived from immature oocytes in vitro. Theriogenology 1994; 42: 527-535. http://dx.doi.org/10.1016/0093-691X(94)90690-K

[9] Tajik $\mathrm{P}$, Shams-Esfandabadi N. In vitro maturation of caprine oocytes in different culture media. Small Rumin Res 2003; 47: 155-158.

http://dx.doi.org/10.1016/S0921-4488(02)00238-9

[10] Funahashi H, Cantley TC, Day BN. Preincubation of cumulus-oocyte complexes before exposure to gonadotropins improves the developmental competence of porcine embryos matured and fertilized in vitro. Theriogenology 1997; 47: 679-686. http://dx.doi.org/10.1016/S0093-691X(97)00026-5

[11] Hinrichs K, Martin MG, Schmidt AL, Friedman PP. Effect of follicular components on meiotic arrest and resumption in horse oocytes. J Reprod Fertil 1995; 104: 149-156. http://dx.doi.org/10.1530/irf.0.1040149

Received on 10-03-2015

Accepted on 12-03-2015

Published on 03-04-2015

\section{DOI: http://dx.doi.org/10.6000/1927-520X.2015.04.01.2}

(C) 2015 Saber Mohamed Abd-Allah; Licensee Lifescience Global.

This is an open access article licensed under the terms of the Creative Commons Attribution Non-Commercial License (http://creativecommons.org/licenses/by-nc/3.0/) which permits unrestricted, non-commercial use, distribution and reproduction in any medium, provided the work is properly cited. 